\title{
Metacognition of agency is reduced in high hypnotic suggestibility
}

Devin B. Terhune ${ }^{1,2}$ \& Love R. A. Hedman ${ }^{1}$

${ }^{1}$ Department of Experimental Psychology, University of Oxford

${ }^{2}$ Department of Psychology, Goldsmiths, University of London

\section{Corresponding author:}

Devin B. Terhune

Department of Psychology

Goldsmiths, University of London

New Cross, London SE14 6NW

Tel: $+44(0) 2070785148$

Fax: +44 (0) 2079197873

Email: d.terhune@gold.ac.uk

\section{Acknowledgments}

This work was supported by a bursary from the Bial Foundation (198/12) and a Marie Skłodowska-Curie Intra-European Fellowship within the 7th European Community Framework Programme to DBT. We thank Mihaela Duta for programming assistance.

\section{Citation}

Terhune, D. B. \& Hedman, L. R. A. (2017). Metacognition of agency is reduced in high hypnotic suggestibility. Cognition, 168, 176-181. 


\section{Abstract}

A disruption in the sense of agency is the primary phenomenological feature of response to hypnotic suggestions but its cognitive basis remains elusive. Here we tested the proposal that distorted volition during response to suggestions arises from poor metacognition pertaining to the sources of one's control. Highly suggestible and control participants completed a motor task in which performance was reduced through surreptitious manipulations of cursor lag and stimuli speed. Highly suggestible participants did not differ from controls in performance or metacognition of performance, but their sense of agency was less sensitive to cursor lag manipulations, suggesting reduced awareness that their control was being manipulated. These results indicate that highly suggestible individuals have aberrant metacognition of agency and may be a valuable population for studying distortions in the sense of agency.

Keywords: agency; hypnosis; hypnotizability; metacognition; schizotypy; volition 


\section{Introduction}

The primary phenomenological feature of a response to a hypnotic suggestion is the perception that one is not the author of one's actions or experience (Oakley \& Halligan, 2013). This disruption of one's sense of agency is reliably observed in highly suggestible (HS) individuals, who comprise $10-15 \%$ of the population (Woody \& Barnier, 2008). The magnitude of these disruptions closely parallels those of patients with schizophrenia (Polito, Langdon, \& Barnier, 2015), indicating that HS individuals may be valuable in identifying the neurocognitive bases of distortions in the sense of agency (Terhune, Cleeremans, Raz, \& Lynn, in press).

The mechanisms underlying response to suggestion and distorted volition among HS individuals are poorly understood but multiple theories converge on the hypothesis that distorted sense of agency during hypnotic responding arises from a disruption of meta-awareness. Different models have proposed that hypnotic responding is driven by cognitive control that is experienced as extra-volition because of a disruption of executive monitoring (Dienes \& Perner, 2007; Hilgard, 1977; Kunzendorf, 1985-86; Miller, Galanter, \& Pribram, 1960) (see also Kirsch \& Lynn, 1998; Spanos, 1986). One theory has specifically hypothesized that responses to suggestion are facilitated by a disruption of meta-awareness of intentions pertaining to one's responses (Dienes \& Perner, 2007).

Multiple lines of evidence offer support for an involvement of aberrant metacognition in high hypnotic suggestibility. Hypnotic suggestibility is negatively associated with mindfulness and meditators display reduced or average hypnotic suggestibility (Semmens-Wheeler \& Dienes, 2012; Spanos, Steggles, Radtke-Bodorik, \& Rivers, 1979). Meditators seem to have greater awareness of motor intentions (Jo, Hinterberger, Wittmann, \& Schmidt, 2015) whereas HS individuals seem to have delayed awareness of such intentions (Lush, Naish, \& Dienes, 2016). Reducing meta-awareness may also enhance suggestibility (Brown, Antonova, Langley, \& Oakley, 2001). Finally, HS individuals display reduced prefrontal activity or prefrontal functional connectivity either at baseline or following a hypnotic induction (Jamieson \& Burgess, 2014; McGeown, Mazzoni, Venneri, \& Kirsch, 2009; Terhune, Cardeña, \& Lindgren, 2011), including in medial prefrontal regions that have been implicated in metacognition of 
agency (Miele, Wager, Mitchell, \& Metcalfe, 2011). Nevertheless, the prediction that metacognition pertaining to one's sense of agency is diminished in HS individuals has not yet been directly tested.

In this study we tested the prediction that high hypnotic suggestibility is characterized by reduced metacognition of agency. HS participants and controls completed a motor control task in which performance was disrupted through surreptitious manipulations related (cursor lag), and unrelated (stimuli speed), to motor control (Metcalfe \& Greene, 2007; Metcalfe, Van Snellenberg, DeRosse, Balsam, \& Malhotra, 2012). They were presented with descending visual stimuli and attempted to capture targets and avoid non-targets using a moving cursor and subsequently judged their performance and control. Previous research showed that sense of agency in patients with schizophrenia was less sensitive to cursor lag manipulations than in controls (Metcalfe et al., 2012), suggesting impaired metacognition of agency. On the basis of the phenomenological similarity of distorted agency in patients with schizophrenia and HS individuals (Polito et al., 2015), we expected that the latter group's sense of agency would be similarly less sensitive to manipulations of cursor lag, but not of stimuli speed, relative to controls.

\section{Methods}

\subsection{Participants}

74 right-handed individuals $\left(M_{\mathrm{Age}}=24.5, S E=0.8 ; 48\right.$ females; years of post-secondary education: $3.6 \pm 0.3$ ) consented to participate in this study in accordance with local ethical approval. We expected an effect size for the primary group difference in the within-participant regression analyses to be similar to that of a previous study on patients with schizophrenia $(d \mathrm{~s}=0.96-1.17$; Metcalfe et al., 2012) and so we made a conservative estimate of $d=0.8$. Assuming a two tailed $\alpha=.05$ and $1-\beta=.90$ and an allocation ratio of 2:1 (approximately twice as many controls as HS participants), this amounted to a required sample size of 62 participants. We collected data past this number to increase statistical power and account for the possible omission of participants (none were omitted from the final sample); we ceased data collection once a certain time point in the academic calendar had been reached. Participants were recruited randomly from a larger sample $(N=617)$ that had previously undergone testing for hypnotic suggestibility. 


\subsection{Materials and Procedure}

Hypnotic suggestibility was measured with the Harvard Group Scale of Hypnotic Susceptibility: Form A (HGSHS:A; Shor \& Orne, 1962), a widely-used, group-administered behavioral measure of hypnotic suggestibility. This scale has been extensively validated (Woody \& Barnier, 2008) and exhibited acceptable internal consistency (Cronbach's $\alpha=.67)$. The scale consists of a relaxation-based hypnotic induction and 12 suggestions of varying difficulty. Representative items include facilitative motor (e.g., arm heaviness), inhibitory motor (e.g., arm paralysis), and inhibitory cognitive (e.g., posthypnotic amnesia) suggestions. Participants rate their responsiveness to each suggestion based on overt behavioral responses and responsiveness to each item is scored dichotomously. Summary scores range from 0 to 12 with scores in the range of 9-12 reflecting high hypnotic suggestibility.

The Metacognition of Agency Task (Metcalfe \& Greene, 2007; Metcalfe et al., 2012) indexed the extent to which participants were aware that their motor control had been manipulated. Each trial consisted of 54 stimuli (the letters $\mathrm{X}$ and $\mathrm{O}$; Arial, white font, font size 20; 27 each) descending from the top to the bottom of the monitor (see Figure 1). A grey horizontal track $(1 \mathrm{~cm}$ tall) that stretched across the monitor $20 \mathrm{~cm}$ from the bottom included a superimposed $1 \times 1 \mathrm{~cm}$ cursor (white square) that was controlled by the mouse. Participants were instructed to capture targets (Xs) using the cursor, whilst avoiding non-targets (Os) and received performance feedback (32ms auditory tone; high pitch [5000Hz] for correct responses; low pitch [1000Hz] for incorrect responses) (for further details, see (Metcalfe, Eich, \& Castel, 2010)). We manipulated two variables: Stimuli speed (8 v. 10 pixels per frame rate [slow v. fast conditions, respectively]) and Cursor lag ( 0 v. 3 v. 6 frame rates of delay of mouse movements [control (0ms) v. short lag (50ms) v. long lag (100ms) conditions, respectively]). Participants were not preinformed about these manipulations. Trials took approximately $28-34 \mathrm{~s}$ to complete. After each trial, participants rated their performance (Judgments of Performance [JOPs]; from poor to perfect) and control (Judgments of Agency [JOAs]; from no control to completely in control) using visual analogue scales $(0$ to 1) on the monitor. 


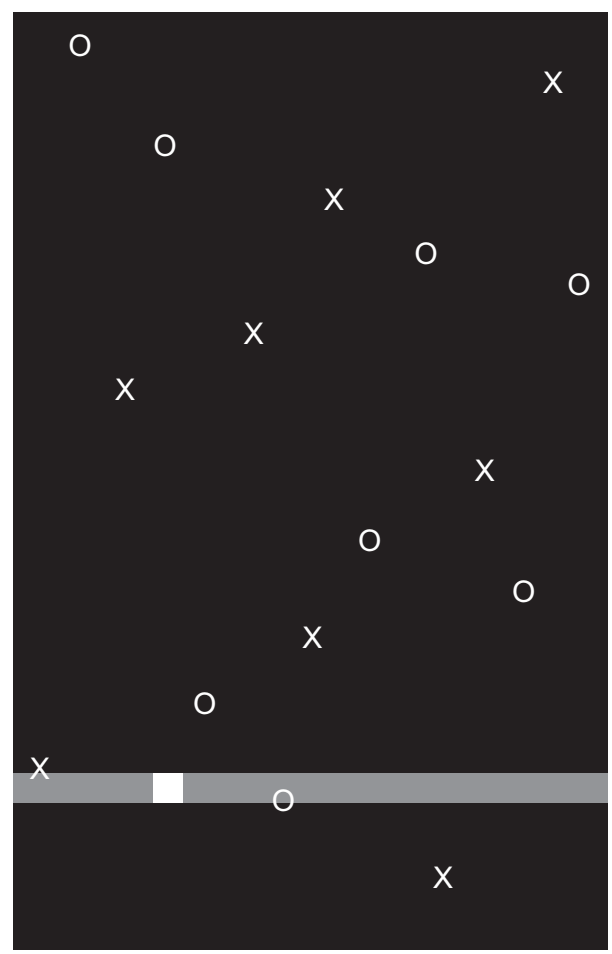

Figure 1. Metacognition of Agency Task (MAT). Participants moved a cursor (white square) along a horizontal track in order to capture descending targets (Xs) and avoid descending non-targets (Os). Stimuli speed and cursor lag were surreptitiously manipulated on different trials to disrupt performance (see Method).

Participants completed the HGSHS:A and MAT on separate days. The experimenter administering the MAT was masked to participants' HGSHS:A scores and participants were naïve to the hypotheses under test, ensuring a double-blind design. Prior to the task, video/computer gaming experience was evaluated on a 7-point Likert scale (1: never, 2: a few times, 3: more than a few times, 4: once in a while, 5: many times, 6: regularly, 7: daily) (data were missing for one participant). The MAT was completed on a PC using a rotatable DELL LED display $(1920 \times 1200$ pixels, $56 \times 36 \mathrm{~cm}$; refresh rate: $60 \mathrm{~Hz})$ at a distance of $70 \mathrm{~cm}$ with the monitor in the vertical orientation. Stimulus presentation and response collection was implemented with Psychtoolbox (Brainard, 1997; Kleiner, Brainard, \& Pelli, 2007) for MATLAB ${ }^{\circledR}$ (2014a, MathWorks, Natick, MA). Participants completed two practice trials followed by 30 experimental 
trials with each combination of Stimuli speed (2 levels) and Cursor lag (3 levels) occurring with random presentation in five blocks of 6 trials. After completing the task, participants were asked whether they had noticed the lag (scored dichotomously: yes or no) and to estimate the frequency of trials on which a lag was present $(0-100 \%)$.

\subsection{Analyses.}

Raw data are available in the Supplementary Material. The dependent variables included performance measures $\left(d^{\prime}\right.$, hit rates [proportion of "caught" targets] and false alarm rates [proportion of "caught" nontargets]) and metacognition measures (JOPs and JOAs). Data were analysed using MATLAB and SPSS ${ }^{\circledR}$ (v. 22, IBM) with 2 (Speed: slow vs. fast) $\times 3$ (Lag: control vs. short vs. long) $\times 2$ (Group: controls vs. HS participants) mixed-model ANOVAs. Greenhouse-Geisser corrections were applied when data violated the assumption of sphericity. Subsidiary analyses used univariate Welch ANOVAs or $t$-tests because of the uneven ratio of participants in the two groups (uncorrected $d f \mathrm{~s}$ are reported). We estimated confidence intervals for effect sizes ( $\eta^{2}$ and robust Cohen's $d$; Algina, Keselman, \& Penfield, 2005) using bootstrap resampling (10,000 samples; bias-corrected and accelerated method; Efron, 1987). Within-participant regression analyses were performed in which JOAs were regressed on $d^{\prime}$, JOPs, Stimuli speed, and Cursor lag (Metcalfe et al., 2012); group comparisons of individual beta coefficients were subsequently done using Welch $t$-tests. On the basis of research suggesting that HS participants constitute a discrete group (Oakman \& Woody, 1996), the primary analyses treated Hypnotic suggestibility as a categorical variable. Nevertheless, we supplement all critical effects with Spearman, point biserial, or partial Spearman correlations in which Hypnotic suggestibility is treated as a continuous variable, computed using the Robust Correlation Toolbox in MATLAB (Pernet, Wilcox, \& Rousselet, 2012); in all cases, the categorical and continuous analyses converge. All analyses were two-tailed.

We also used Bayesian statistics to contrast group differences (HS participants - controls [ $\Delta \mathrm{HS}]$ ) with those between patients with schizophrenia and controls (patients - controls [ $\Delta \mathrm{SZ}]$; Metcalfe et al., 2012) or other group differences in our data. In particular, we examined: 1) whether $\Delta \mathrm{HS}$ in JOPs differed from 
$\Delta \mathrm{SZ}$ in JOPs; 2) whether $\Delta \mathrm{HS}$ in JOAs differed from $\Delta \mathrm{HS}$ in JOPs; and 3) whether $\Delta \mathrm{HS}$ in cursor lag beta coefficients (predicting JOAs) differed from $\Delta \mathrm{SZ}$ beta coefficients. Toward this end, we computed Bayes factors (BFs) in MATLAB (Dienes, 2008, 2011) for group differences using $\Delta$ SZ or $\Delta H S$ values as priors. BFs index the likelihood that data favor the alternative or null hypothesis and are thereby especially informative in the interpretation of non-significant $p$-values from orthodox statistical tests (Dienes, 2014). The theoretical range of BFs extends from 0 to $\infty$, with values less than or greater than 1 reflecting evidence for or against the null hypothesis, respectively. BFs less than 0.33 are typically interpreted as providing substantial evidence for the null hypothesis, those greater than 3 as providing substantial evidence for the alternative, and those between 0.33 and 3 as being insensitive in distinguishing between the two (Dienes, 2011; Jeffreys, 1961). In each case, BFs were calculated with the group effect ( $\Delta \mathrm{HS}$ : $M \pm S E$ ) contrasted against the respective prior specified as a normal distribution, $\mathrm{BF}_{\mathrm{N}(M, S D)}$, where $M=\Delta$ and $S D=S E(\Delta)$ (the use of a default $S D=M / 2$ yielded comparable results in all but one case), because a hypothesis maintaining that the effects are comparable predicts that near-zero values are unlikely (Dienes, 2014).

\section{Results}

\subsection{Demographic information}

HGSHS:A scores ranged from 0 to 11 , with a mean $( \pm S E)$ of $6.4 \pm 0.3$, thereby encompassing a broad range of levels of hypnotic suggestibility. We contrasted HS participants ( $n=16$; HGSHS:A: 9.50 \pm 0.18 ) against the remainder of the sample (henceforth controls; $n=58$; HGSHS:A: $5.52 \pm 2.24$ ). The two groups did not significantly differ in age (controls: $24.5 \pm 0.3$; HS: $24.4 \pm 0.4), t(72)=0.03, p=.98, d=0.13[-0.23$, 0.48], gender (female:male ratios; controls: 39:19; HS: 9:7), $\chi^{2}(1)=0.67, p=0.42, \varphi=.09[.00, .31]$, years of post-secondary education (controls: $3.7 \pm 0.3$; HS: $2.9 \pm 0.4$ ), $t(72)=1.44, p=.16, d=0.38[0.05,0.72]$, or video/computer gaming experience (controls: $2.4 \pm 0.2$; HS: $2.4 \pm 0.4), t(71)=0.17, p=.86, d=0.19[-0.11$, $0.51]$. 


\subsection{Task performance}

A 2 (Speed) $\times 3(\mathrm{Lag}) \times 2($ Group $)$ mixed-model ANOVA on $d^{\prime}$ values revealed large main effects of

Speed, $F(1,72)=232.42, p<.001, \eta_{\mathrm{p}}{ }^{2}=.76[.66, .82]$, and Lag, $F(2,144)=261.19, p<.001, \eta_{\mathrm{p}}{ }^{2}=.78[.72, .82]$, reflecting declines in performance as the magnitude of each manipulation increased (see Figure 2a). In addition, there was a small Speed $\times$ Lag interaction, $F(2,144)=3.86, p=.023, \eta_{\mathrm{p}}{ }^{2}=.05[.00, .13]$, reflecting a greater detrimental influence of Speed on performance in the short lag condition $\left(\eta^{2} \mathrm{~s}\right.$ : control: .63 [.49, $.72]$, short: .68 [.55, .75], long: .65 [.51,.73]). By contrast, there was no significant effect of Group, $F(1,72)=0.02, p=.88, \eta_{\mathrm{p}}{ }^{2}<.01[.00, .01], r_{\mathrm{s}}=.07, p=.58[-.17, .29]$, or any other significant effects, $F_{\mathbf{S}}<1.7$, $p \mathrm{~s}>.2, \eta_{\mathrm{p}}{ }^{2} \mathbf{s}<.03$.

The analysis of hit rates similarly revealed large main effects of Speed, $F(1,72)=508.37, p<.001$, $\eta_{\mathrm{p}}{ }^{2}=.88[.82, .91]$, and Lag, $F(2,144)=563.88, p<.001, \eta_{\mathrm{p}}{ }^{2}=.89[.85, .91]$, which were qualified by a small Speed $\times$ Lag interaction, $F(2,144)=5.98, p=.003, \eta_{\mathrm{p}}{ }^{2}=.08[.01, .16]$, reflecting a decline in the influence of Speed as Lag increased $\left(\eta^{2}\right.$ s: control: $.85[.78, .88]$, short: .80 [.72, .85], long: .72 [.60,.78]), but no other significant effects, $F_{\mathbf{S}}<1.1, p \mathrm{~s}>.35, \eta_{\mathrm{p}}{ }^{2} \mathbf{s}<.02$ (see Figure $2 \mathbf{b}$ ). By contrast, there were no significant effects on false alarm rates, $F \mathbf{s}<2.3, p \mathbf{s}>.11$, which were uniformly low (see Figure 2c). Neither hit rates, $r_{\mathrm{s}}=.05$, $p=.70[-.18, .26]$, nor false alarm rates, $r_{\mathrm{s}}=-.03, p=.78[-.26, .20]$, significantly correlated with Hypnotic suggestibility. These analyses demonstrate that performance declined with increased cursor lag, as previously observed (Metcalfe \& Greene, 2007; Metcalfe et al., 2012), and stimuli speed, but that performance was not significantly related to hypnotic suggestibility. 

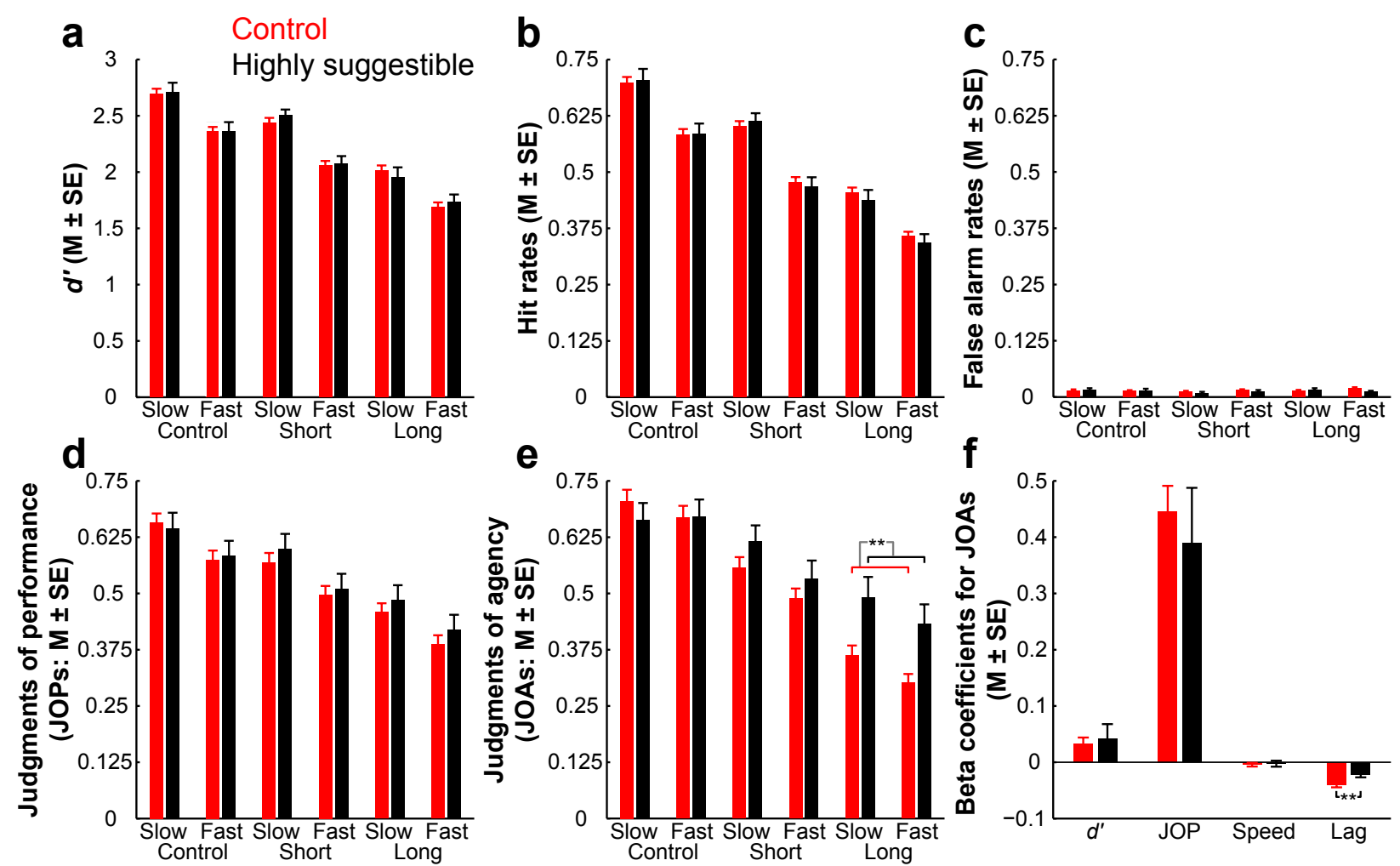

Figure 2. Performance and metacognitive judgments in the Metacognition of Agency Task (MAT). (a) $d^{\prime}$,

(b) hit rates, (c) false alarm rates, (d) Judgments of Performance (JOPs), and (e) Judgments of Agency (JOAs) as a function of Stimuli speed (slow v. fast), Cursor lag (control v. short v. long), and Group (control v. highly suggestible [HS]). (e) Beta coefficients for individual predictors ( $d^{\prime}$, JOP, Stimuli speed, and Cursor lag) in within-participant regression analyses on JOAs as a function of Group. $* * p<.01$

\subsection{Judgments of Performance (JOPs)}

As can be seen in Figure 2d, there were large main effects of Speed, $F(1,72)=91.76, p<.001, \eta_{\mathrm{p}}{ }^{2}=.56[.40$, $.66]$ and Lag, $F(2,144)=145.35, p<.001, \eta_{\mathrm{p}}{ }^{2}=.67[.58, .73]$, on JOPs, reflecting self-perceived performance declines with increases in the magnitude of experimental manipulations, but no significant effect of Group, $F(1,72)=0.18, p=.68, \eta_{\mathrm{p}}{ }^{2}<.01[.00, .07], r_{\mathrm{s}}=.12, p=.12[-.07, .41]$, and no other significant effects, $F_{\mathrm{s}}<1.3, p \mathrm{~s}>.27, \eta_{\mathrm{p}}{ }^{2}<<.02$. Bayesian analyses testing the hypothesis that JOP group differences $(\Delta \mathrm{HS}$ :

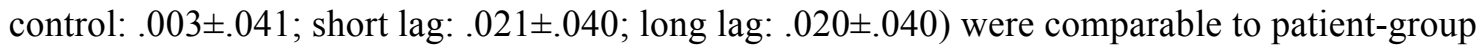


differences $(\Delta \mathrm{SZ})$ (Metcalfe et al., 2012) yielded clear evidence for the null hypothesis (control: $\mathrm{BF}_{\mathrm{N}(.08 \text {, }}$ $.06)=0.32$; short lag: $\mathrm{BF}_{\mathrm{N}(.11, .05)}=0.27$; long lag: $\left.\mathrm{BF}_{\mathrm{N}(.12,05)}=0.21\right)$. This indicates that group differences in JOPs were lower than those between patients and controls and suggest that HS participants' JOPs were dissimilar from those of patients with schizophrenia. These results suggest that JOPs closely parallel behavioral performance patterns and do not relate to Hypnotic suggestibility.

\subsection{Judgments of Agency (JOAs)}

JOAs similarly declined with task manipulations (Figure 2e), as reflected in main effects of Speed, $F(1,72)=43.37, p<.001, \eta_{\mathrm{p}}{ }^{2}=.38[.20, .51]$, and Lag, $F(2,144)=119.91, p<.001, \eta_{\mathrm{p}}{ }^{2}=.63[.53, .69]$, which were qualified by a Speed $\times$ Lag interaction, $F(2,144)=5.32, p=.006, \eta_{\mathrm{p}}{ }^{2}=.07[.01, .15]$, which reflected a smaller effect of Speed in the control condition $\left(\eta_{\mathrm{p}}{ }^{2} \mathrm{~s}\right.$ : control: .10 [.01, .24], short: .34 [.17, .48], long: .27 $[.11, .42])$. The effect of Speed on JOAs replicates a previous study observing the same effect (Vuorre \& Metcalfe, 2016). The Lag effect was further moderated by the predicted Lag $\times$ Group interaction, $F(2,144)=8.57, p=.001, \eta_{\mathrm{p}}{ }^{2}=.11[.02, .20]$. Relative to controls, HS participants reported greater JOAs in the long lag condition, $F(1,72)=7.57, p=.012, \eta^{2}=.11[.01, .25], r_{\mathrm{s}}=.43, p<.001[.20, .61]$, but the two groups did not significantly differ in the control, $F(1,72)=0.20, p=.66, \eta^{2}=.00[.00, .07], r_{\mathrm{s}}=-.06, p=.59[-$ $.30, .19]$, or in the short lag, $F(1,72)=1.55, p=.22, \eta^{2}=.02[.00, .11], r_{\mathrm{s}}=.19, p=.10[-.04, .40]$, conditions. All other effects were non-significant, $F_{\mathrm{s}}<1.7, p \mathrm{~s}>.2, \eta_{\mathrm{p}}{ }_{\mathrm{s}} \mathrm{s}<.03$.

We next examined the specificity of the differential lag effect across groups on JOAs, namely whether it was different from the corresponding non-significant group effect on JOPs. Toward this end, we repeated the analyses on Judgments, collapsing across Speeds and including Judgment type (JOP v. JOA) as a repeated-measures independent variable. This analysis replicated the foregoing effects but also revealed a Judgment type $\times$ Lag $\times$ Group interaction, $F(2,144)=7.46, p=.002, \eta_{\mathrm{p}}{ }^{2}=.09[.02, .18]$. The Judgment type $\times$ Group interaction, reflecting a greater Group discrepancy in the difference between JOAs and JOPs, was restricted to the long lag condition (control: $F(1,72)=0.30, p=.58, \eta_{\mathrm{p}}{ }^{2}<.01[.00, .08]$; short lag: $F(1,72)=0.72, p=.40, \eta_{\mathrm{p}}{ }^{2}=.01[.00, .10]$; long lag: $\left.F(1,72)=8.86, p=.004, \eta_{\mathrm{p}}{ }^{2}=.11[.01, .25]\right)$. This 
indicates that the difference between JOAs and JOPs was larger in HS participants, but only in the long lag condition. Bayesian analyses contrasting group differences in JOAs ( $\triangle \mathrm{HS}-\mathrm{JOA}$ ) against the corresponding difference in JOPs $(\Delta \mathrm{HS}-\mathrm{JOP})$ further corroborated these differential effects. Although the data in the control, $-.020 \pm .044, \mathrm{BF}_{\mathrm{N}(.003, .041)}=0.75$, and short lag, $.051 \pm .041, \mathrm{BF}_{\mathrm{N}(.021, .040)}=1.35$, conditions

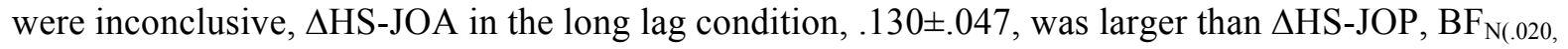
$.040)=7.13$. Thus, the magnitude of JOA group differences was larger than that for JOP group differences but only in the long lag condition. These results indicate that JOAs declined with increased task manipulations, in a parallel fashion to JOPs and behavioural performance, but that HS participants reported selectively greater control, but not performance, in the high cursor lag condition, when performance was lowest. This suggests that HS participants' sense of agency is less sensitive to manipulations of control.

\subsection{Regression analyses}

We next sought to determine whether poorer metacognition of agency in HS participants was independent of performance and metacognition of performance. We expected that even when performance, JOPs, and Stimuli speed were considered, cursor lag manipulations would still have less influence on JOAs in HS participants, as previously observed in patients with schizophrenia (Metcalfe et al., 2012). To test this hypothesis, we submitted data to within-participant regression analyses in which JOAs were regressed on $d^{\prime}$, JOPs, Stimuli speed, and Lag (Metcalfe, Eich, \& Miele, 2013; Metcalfe et al., 2012). Across groups, mean (unstandardized) beta coefficients were significantly different from 0 for $d^{\prime}(.03 \pm .01), t(73)=3.33$, $p=.001, d=0.39[0.160 .63]$, JOPs $(.43 \pm .04), t(73)=10.35, p<.001, d=1.20[0.93,1.58]$, and Lag $(-$ $.04 \pm .003), t(73)=10.63, p<.001, d=1.24[1.04,1.51]$, thereby replicating both the magnitude and direction of these coefficients as observed in previous studies (Metcalfe et al., 2013; Metcalfe et al., 2012). In contrast, beta coefficients for Stimuli speed did not significantly differ from $0(-.004 \pm .003), t(73)=1.42$, $p=.16, d=0.16[-0.06,0.40]$. These results indicate that JOAs were higher when $d^{\prime}$ and JOPs were higher, but lower when the Lag was longer, and were not reliably related to Stimuli speed. 
We expected that Lag beta coefficients would be closer to 0 in HS participants than in controls, indicating that their sense of agency is less sensitive to manipulations of motor control, but that the two groups would not exhibit differential betas for the other predictors (Metcalfe et al., 2012). In support of this prediction, Lag beta coefficients were closer to 0 in HS participants than in controls, $t(72)=2.93$, $p=.006, d=0.67[0.40,0.98], r_{\mathrm{s}}=.32, p=.006[.09, .50]$, (see Figure 2f), whereas the two groups did not significantly differ in beta coefficients for $\left.d^{\prime}, t(72)=0.31, p=.76, d=0.25[-0.17,0.67]\right), r_{\mathrm{s}}=.08, p=.49[-.16$, $.31]$, JOPs, $t(72)=0.51, p=.61, d=0.06[-0.32,0.44]), r_{\mathrm{s}}=.05, p=.65[-.17, .27]$, or Stimuli speed, $t(72)=0.29, p=.77, d=0.05[-0.29,0.38]), r_{\mathrm{s}}=-.06, p=.61[-.28, .16]$. Bayesian analyses testing the hypothesis that the group difference $(\Delta \mathrm{HS})$ in lag beta coefficients $(.019 \pm .006)$ was similar to two corresponding patient-control differences $(\Delta$ SZ) (Metcalfe et al., 2012) yielded support for the null hypothesis for one difference ( $\Delta \mathrm{SZ}$ : short lag vs. control; $\left.\mathrm{BF}_{\mathrm{N}(.25, .06)}=0.01\right)$ but insensitive results for the second difference $\left(\Delta \mathrm{SZ} \text { : long lag vs. control; } \mathrm{BF}_{\mathrm{N}(1.17,08)}=1.91\right)^{1}$. These results indicate that $\mathrm{HS}$ participants' sense of agency is selectively less sensitive to cursor lag manipulations, suggesting impaired metacognition of agency, although the data were insensitive in assessing whether this group difference was of comparable magnitude to those observed between patients with schizophrenia and controls.

\subsection{Lag awareness and estimated frequency}

Ninety-two percent of participants detected the cursor lag, with no significant group differences (controls: 91\%; HS participants: 94\%; Fisher's exact $p=1.0, \varphi=0.04[.00, .11], r_{\mathrm{s}}=.03, p=.83[-.19, .27]$. Across groups, participants underestimated the frequency of the lag $(46 \% \pm 2.5$; objective: $67 \%, t=8.30, p<.001$, $d=0.97[.75,1.24])$, with estimated lag frequency not significantly differing between groups (controls: $45 \% \pm 2.9$; HS participants: $52 \% \pm 4.4), t(72)=1.34, p=.19, d=0.20[-0.10,0.52], r_{\mathrm{s}}=-.11, p=.36[-.33, .13]$. In order to determine whether these variables influenced metacognition of agency, we repeated the between-group contrast of Lag beta coefficients in an ANCOVA including Estimated lag frequency as a

\footnotetext{
${ }^{1}$ Our results are not directly comparable to those of Metcalfe et al. (2012) and thus these results should be interpreted with caution: Metcalfe et al. (2012) dummy-coded the two different lags and treated them as two binary predictors whereas we included lag as a single 3-level categorical predictor.
} 
covariate. The main effect of Group remained significant, $F(1,71)=7.78, p=.007, \eta_{\mathrm{p}}{ }^{2}=.10[.01, .24]$, $r_{\mathrm{ps}}=.30, p=.010,[.06, .50]$, replicating the result that HS participants' beta coefficients were nearer to 0 than controls. In addition, there was a comparably-sized main effect of Estimated lag frequency, $F(1,71)=7.75, p=.007, \eta_{\mathrm{p}}{ }^{2}=.10[.01, .24]$, reflecting a negative association, $r_{\mathrm{s}}=-.30, p=.009[-.50,-.08]$. These data indicate that metacognition of agency was independently lower in HS participants and in those who underestimated the frequency of the lag.

\section{Discussion}

Highly suggestible individuals did not differ from controls in motor control or in metacognition of performance but reported greater sense of agency when control was disrupted by a temporal lag on their motor output. Further analyses demonstrated that highly suggestible individuals' sense of agency was less predictable from the cursor lag than that of controls and that this group difference was independent of a number of relevant parameters including behavioural performance, metacognition of performance, manipulation of stimuli speed, and estimated lag frequency. In addition, the effects were stable irrespective of whether or not hypnotic suggestibility was treated as a binary or continuous measure. These results indicate that highly suggestible individuals' sense of agency is less sensitive to manipulations of their perceived control and thereby support the proposal that high hypnotic suggestibility is characterized by a disruption of meta-awareness (Dienes \& Perner, 2007; Hilgard, 1977; Kunzendorf, 1985-86; Spanos, 1986). In particular, highly suggestible individuals may have failed to detect the impact of the lag on their control because of a delay in their awareness of when they initiated their motor responses (Lush et al., 2016). Impaired metacognition may facilitate hypnotic suggestibility by reducing accessibility of intentions associated with responses to suggestions (Dienes \& Perner, 2007), giving rise to the perceived loss of authorship over one's responses (Bowers, 1981; Polito et al., 2015; Walsh, Oakley, Halligan, Mehta, \& Deeley, 2015; Weitzenhoffer, 1974). 
Highly suggestible individuals displayed the same pattern of aberrant metacognition of agency as patients with schizophrenia (Metcalfe et al., 2012), thus complementing phenomenological data showing nearly equivalent levels of distorted volition in these groups during response to suggestion and passivity symptoms, respectively (Polito et al., 2015). Relative to controls, insensitivity to lag manipulations was smaller in highly suggestible individuals $(d=0.67)$ than in patients with schizophrenia $(d \mathrm{~s}=0.96-1.17$; Metcalfe et al., 2012); however, Bayesian analyses indicated that the available data were inconclusive regarding whether these effects are comparable, thereby precluding any firm conclusions regarding their (dis)similarity (Dienes, 2014). Moreover, qualitative effect size differences might be attributable to the longer cursor lags in the latter study (250-500ms; Metcalfe et al., 2012) compared to those in the current study (50-100ms). Patients with schizophrenia also significantly overestimated their performance compared with controls, whereas highly suggestible individuals did not; these results suggest that patients exhibit a broad deficit in metacognition whereas aberrant metacognition in highly suggestible individuals may be specific to the sense of agency. Further independent evidence for differences between these groups comes from data showing that patients with schizophrenia display attenuated or normal hypnotic suggestibility (Frischholz, Lipman, Braun, \& Sachs, 1992) and that the majority of highly suggestible individuals have a healthy cognitive profile (Parris, 2017; Terhune \& Cardeña, 2015). Nevertheless, the shared response patterns of these two groups indicate that highly suggestible individuals may provide a valuable model for distortions in the sense of agency (Polito et al., 2015; Terhune et al., in press; Walsh et al., 2015) and are consistent with preliminary research linking hypnotic suggestibility to schizotypy (Connors et al., 2014; Gruzelier et al., 2004), which is similarly characterized by aberrant sense of agency (Moore \& Bravin, 2015). Further research is required to characterize the associations between schizotypy and hypnotic suggestibility and the extent to which distortions in the sense of agency in these different populations have distinct and shared mechanisms. 


\section{References}

Algina, J., Keselman, H. J., \& Penfield, R. D. (2005). An alternative to Cohen's standardized mean difference effect size: A robust parameter and confidence interval in the two independent groups case. Psychol Methods, 10, 317-328.

Bowers, K. S. (1981). Do the Stanford Scales tap the "classic suggestion effect"? International Journal of Clinical and Experimental Hypnosis, 29(1), 42-53. doi: 10.1080/00207148108409142

Brainard, D. H. (1997). The Psychophysics Toolbox. Spatial Vision, 10(4), 433-436.

Brown, R. J., Antonova, E., Langley, A., \& Oakley, D. A. (2001). The effects of absorption and reduced critical though on suggestibility in an hypnotic context. Contemporary hypnosis, 18, 62-72.

Connors, M. H., Halligan, P. W., Barnier, A. J., Langdon, R., Cox, R. E., Elliotta, J., . . Coltheart, M. (2014). Hypnotic analogues of delusions: The role of delusion proneness and schizotypy. Personality and Individual Differences, 57, 48-53.

Dienes, Z. (2008). Understanding psychology as a science: An introduction to scientific and statistical inference. Basingstoke: Palgrave Macmillan.

Dienes, Z. (2011). Bayesian versus orthodox statistics: Which side are you on? Perspectives on Psychological Science, 6(3), 274-290. doi: 10.1177/1745691611406920

Dienes, Z. (2014). Using Bayes to get the most out of non-significant results. Frontiers in psychology, 5, 781. doi: 10.3389/fpsyg.2014.00781

Dienes, Z., \& Perner, J. (2007). Executive control without conscious awareness: The cold control theory of hypnosis. In G. A. Jamieson (Ed.), Hypnosis and conscious states: The cognitive neuroscience perspective (pp. 293-314). Oxford, UK: Oxford University Press.

Efron, B. (1987). Better bootstrap confidence intervals. Journal of the American Statistical Association, $82,171-185$.

Frischholz, E. J., Lipman, L. S., Braun, B. G., \& Sachs, R. G. (1992). Psychopathology, hypnotizability, and dissociation. American Journal of Psychiatry, 149(11), 1521-1525. 
Gruzelier, J., De Pascalis, V., Jamieson, G., Laidlaw, T., Naito, A., Bennett, B., \& Dwivedi, P. (2004). Relations between hypnotizability and psychopathology revisited. Contemporary hypnosis, 21, 169-176.

Hilgard, E. R. (1977). The problem of divided consciousness: A neodissociation interpretation. Annals of the New York Academy of Sciences, 296, 48-59.

Jamieson, G. A., \& Burgess, A. P. (2014). Hypnotic induction is followed by state-like changes in the organization of EEG functional connectivity in the theta and beta frequency bands in highhypnotically susceptible individuals. Front Hum Neurosci, 8, 528. doi:

10.3389/fnhum.2014.00528

Jeffreys, H. (1961). The theory of probability (3rd ed.). Oxford, UK: Oxford University Press.

Jo, H. G., Hinterberger, T., Wittmann, M., \& Schmidt, S. (2015). Do meditators have higher awareness of their intentions to act? Cortex, 65, 149-158. doi: 10.1016/j.cortex.2014.12.015

Kirsch, I., \& Lynn, S. J. (1998). Social-cognitive alternatives to dissociation theories of hypnotic involuntariness. Review of General Psychology, 2(1), 66-80.

Kleiner, M., Brainard, D., \& Pelli, D. (2007). What's new in Psychtoolbox-3? Perception 36 ECVP Abstract Supplement. doi: 10.1068/v070821

Kunzendorf, R. G. (1985-86). Hypnotic hallucinations as 'unmonitored' images. Imagination, Cognition, and Personality, 5, 255-270.

Lush, P., Naish, P., \& Dienes, Z. (2016). Metacognition of intentions in mindfulness and hypnosis. Neuroscience of Consciousness, 1-10.

McGeown, W. J., Mazzoni, G., Venneri, A., \& Kirsch, I. (2009). Hypnotic induction decreases anterior default mode activity. Consciousness and Cognition, 18(4), 848-855. doi: 10.1016/j.concog.2009.09.001

Metcalfe, J., Eich, T. S., \& Castel, A. D. (2010). Metacognition of agency across the lifespan. Cognition, 116(2), 267-282. doi: 10.1016/j.cognition.2010.05.009 
Metcalfe, J., Eich, T. S., \& Miele, D. B. (2013). Metacognition of agency: Proximal action and distal outcome. Exp Brain Res, 229(3), 485-496. doi: 10.1007/s00221-012-3371-6

Metcalfe, J., \& Greene, M. J. (2007). Metacognition of agency. Journal of Experimental Psychology: General, 136(2), 184-199. doi: 10.1037/0096-3445.136.2.184

Metcalfe, J., Van Snellenberg, J. X., DeRosse, P., Balsam, P., \& Malhotra, A. K. (2012). Judgements of agency in schizophrenia: An impairment in autonoetic metacognition. Philosophical Transactions of the Royal Society of London B: Biological Sciences, 367(1594), 1391-1400. doi:

$10.1098 /$ rstb.2012.0006

Miele, D. B., Wager, T. D., Mitchell, J. P., \& Metcalfe, J. (2011). Dissociating neural correlates of action monitoring and metacognition of agency. Journal of Cognitive Neuroscience, 23(11), 3620-3636. doi: 10.1162/jocn_a_00052

Miller, G. A., Galanter, E., \& Pribram, K. H. (1960). Plans and the structure of behavior. New York, NY: Henry Holt \& Co.

Moore, J. W., \& Bravin, J. (2015). Schizotypy and awareness of intention: Variability of W judgments predicts schizotypy scores. Psychology of Consciousness: Theory, Research, and Practice, 2(3), 283-290.

Oakley, D. A., \& Halligan, P. W. (2013). Hypnotic suggestion: Opportunities for cognitive neuroscience. Nature Reviews Neuroscience, 14(8), 565-576. doi: 10.1038/nrn3538

Oakman, J. M., \& Woody, E. Z. (1996). A taxometric analysis of hypnotic susceptibility. Journal of Personality and Social Psychology, 71(5), 980-991. doi: Doi 10.1037/0022-3514.71.5.980

Parris, B. A. (2017). The Role of Frontal Executive Functions in Hypnosis and Hypnotic Suggestibility. Psychology of Consciousness: Theory, Research, and Practice, 4(2), 211-229. doi: $10.1037 /$ cns 0000106

Pernet, C. R., Wilcox, R., \& Rousselet, G. A. (2012). Robust correlation analyses: False positive and power validation using a new open source matlab toolbox. Frontiers in psychology, 3, 606. doi: 10.3389/fpsyg.2012.00606 
Polito, V., Langdon, R., \& Barnier, A. J. (2015). Sense of agency across contexts: Insights from schizophrenia and hypnosis. Psychology of Consciousness: Theory, Research, and Practice, 2(3), 301-314.

Semmens-Wheeler, R., \& Dienes, Z. (2012). The contrasting role of higher order awareness in hypnosis and meditation. Journal of Mind-Body Regulation, 2(1), 43-57.

Shor, R. E., \& Orne, E. C. (1962). Harvard Group Scale of Hypnotic Susceptibility, Form A. Palo Alto, CA: Consulting Psychologists Press.

Spanos, N. P. (1986). Hypnotic behavior: A social psychological interpretation of amnesia, analgesia and trance logic. Behavioral and Brain Sciences, 9(3), 449-467.

Spanos, N. P., Steggles, S., Radtke-Bodorik, H. L., \& Rivers, S. M. (1979). Nonanalytic attending, hypnotic susceptibility, and psychological well-being in trained meditators and nonmeditators. $J$ Abnorm Psychol, 88(1), 85-87.

Terhune, D. B., \& Cardeña, E. (2015). Dissociative subtypes in posttraumatic stress disorders and hypnosis: Neurocognitive parallels and clinical implications. Current Directions in Psychological Science, 24, 452-457. doi: 10.1177/0963721415604611

Terhune, D. B., Cardeña, E., \& Lindgren, M. (2011). Differential frontal-parietal phase synchrony during hypnosis as a function of hypnotic suggestibility. Psychophysiology, 48(10), 1444-1447. doi: 10.1111/j.1469-8986.2011.01211.x

Terhune, D. B., Cleeremans, A., Raz, A., \& Lynn, S. J. (in press). Hypnosis and top-down regulation of consciousness. Neuroscience and Biobehavioral Reviews. doi: 10.1016/j.neubiorev.2017.02.002

Vuorre, M., \& Metcalfe, J. (2016). The relation between the sense of agency and the experience of flow. Conscious Cogn, 43, 133-142. doi: 10.1016/j.concog.2016.06.001

Walsh, E., Oakley, D. A., Halligan, P. W., Mehta, M. A., \& Deeley, Q. (2015). The functional anatomy and connectivity of thought insertion and alien control of movement. Cortex, 64, 380-393. doi: 10.1016/j.cortex.2014.09.012 
Weitzenhoffer, A. M. (1974). When is an "instruction" an "instruction"? Int J Clin Exp Hypn, 22(3), 258269. doi: $10.1080 / 00207147408413005$

Woody, E. Z., \& Barnier, A. J. (2008). Hypnosis scales for the twenty-first century: What do we know and how should we use them? In M. Nash \& A. J. Barnier (Eds.), The Oxford handbook of hypnosis: Theory, research and practice (pp. 255-281). Oxford, UK: Oxford University Press. 